1."J Duncan, ' $\mathrm{G}$ Sebepos-Rogers, ${ }^{2} \mathrm{O}$ Poole-Wilson, ${ }^{3} \mathrm{C}$ To, ${ }^{4} \mathrm{~J}$ B Canavan, ${ }^{1} \mathrm{M}$ Ward, ${ }^{1} \mathrm{~V}$ Kariyawasam, 'R Goel, ' ${ }^{1} \mathrm{~K}$ Patel, 'A Stanton, 'M Sastrillo, 'S Anderson, ' $\mathrm{K}$ Taylor, ' $\mathrm{J}$ Sanderson, 'P Irving. 'Gastroenterology, Guy's \& St Thomas' NHS Foundation Trust; ${ }^{2}$ South London Healthcare Trust, London, UK; ${ }^{3}$ Australian National University, Canberra, Australia; ${ }^{4}$ Kings College London, London, UK

Introduction The prevalence of faecal incontinence (FI) in people with inflammatory bowel disease (IBD) has not been fully explored. FI is not only associated with social stigma but also with decreased quality of life. In the general population prevalence is estimated at between 1-10\%. Awareness of the prevalence of FI in IBD is important to aid management strategies and resource allocation.

Methods Aim: To investigate the prevalence of FI in adults with IBD in a tertiary care setting. Methods: We performed a cross sectional questionnaire survey of 380 adults attending IBD outpatients at Guy's \& St.Thomas' Hospitals. Patient surveys were: the validated International Consultation on Incontinence - Bowels (ICIOB) questionnaire, detailing frequency and severity of bowel pattern, control and quality of life; and the non-validated Bowel Leakage questionnaire, detailing any prior interventions by health care professionals. Demographics of age, gender, diagnosis, Montreal classification, St Mark's Continence Score and disease activity were also recorded. Data was entered into a database and analysed using a SPSS statistical package.

Results Median age was 38 years (IOR 31-50) and 180/380 $(47 \%)$ were female. The mean duration of IBD diagnosis was 8.7 years (3.4-15.1). 151/380 (40\%) had UC vs 229/380 (60\%) CD. Overall, $255 / 380(67 \%)$ reported FI as defined by any episode of uncontrolled bowel opening in the preceding three months, while $343 / 380(90 \%)$ reported anal incontinence of flatus or faeces. Incontinence was strongly associated with disease activity, occurring during disease flares in $57 \%$ of people. However, $37 \%$ experienced incontinence both during relapse and remission, whilst only $5 \%$ experienced incontinence uniquely when in remission. The ICIO-B control score was associated with current disease activity in CD $(r=0.29, p<0.0001)$ but not in UC. There was no significant difference in FI prevalence between patients with Crohn's Disease (CD) or Ulcerative Colitis (UC), (66\% vs $68 \%$, $\mathrm{p}=0.74)$

Conclusion Faecal incontinence in IBD increases in proportion to disease activity. Given the availability of specialist FI interventions and support, we recommend that sensitive questioning regarding FI should be part of routine disease surveillance in the outpatient setting to cater for this unmet need.

Disclosure of Interest None Declared.

\section{PWE-081 THIOGUANINE REMAINS AN ACCEPTABLE ALTERNATIVE IMMUNOSUPPRESSANT IN IBD}

doi:10.1136/gutjnl-2013-304907.370

1." K V Patel, 'R M Goel, ' $\mathrm{V}$ C Kariyawasam, 1 P A Blaker, 'M G Ward, ${ }^{2}$ S Mashari, ${ }^{2}$ A Clark, 'M Sastrillo, 'J Duncan, 'S H Anderson, 'P M Irving, 'J D Sanderson. 'Gastroenterology; 2Pharmacy, Guy's and St Thomas' NHS Foundation Trust, London, UK

Introduction A significant proportion of inflammatory bowel disease (IBD) patients do not tolerate conventional thiopurines (azathioprine (AZA) and mercaptopurine (MP)). Thioguanine (TG) remains an alternative immunomodulator (IM) and has been in regular use in the IBD clinic at Guy's \& St. Thomas' for more than 10 years. Concerns remain regarding nodular regenerative hyperplasia (NRH) in long-term use.

Methods We aimed to describe the indications, efficacy, monitoring and sequelae of TG use in IBD patients. Patients prescribed TG for IBD from 2008-2012, subsequent to a previously published series, were reviewed.
Results 37 patients were identified (15 male, mean age 43 years). 29 patients had Crohn's disease with 2 having co-existing orofacial granulomatosis, 6 had ulcerative colitis and 1 had IBD-unclassified. All had previous exposure to AZA and/or MP. The indications for TG were thiopurine intolerance in 23 and thiopurine induced pancreatitis in 13 patients.

Median duration of exposure was 11 months (IOR: 3-30). Maintenance doses were $20 \mathrm{mg}$ or $40 \mathrm{mg}$. 20 patients (54\%) were in clinical remission at the end of follow-up period, and $16(41 \%)$ of these were steroid free. Mean TGN levels were $1177 \mathrm{pmol} / 8 \times 10^{8} \mathrm{RBC}$ (range $\left.70-3182 \mathrm{pmol} / 8 \times 10^{8}\right)$. Differences in TGN concentrations in clinical responders and non-responders were not statistically significant.

Treatment was withdrawn in 7 patients. Withdrawal was for adverse events in 3 patients, which resolved on TG withdrawal (neutropenic sepsis, pancytopenia and AST rise) and treatment failure in further 3 patients. TG was ceased in another after the diagnosis of metastatic breast cancer whilst receiving Adalimumab and concomitant TG for 12 months. 7 patients discontinued TG because of side effects occurring within 2 months of initiation.

11 patients had long-term exposure (> 18 months). Screening with liver biopsy and/or MRI-Liver was performed in 9 patients. All patients were screened with regular platelet counts and liver profile. None were diagnosed with NRH during follow-up.

Conclusion TG is a viable second-line IM and appears to be effective with an acceptable safety profile. Side effects are uncommon, occur early and cease upon TG withdrawal. Chief indications are previous failure of immunosuppressant therapy or thiopurine-associated pancreatitis. There were no cases of TG-associated pancreatitis. Concerns regarding hepatotoxicity and NRH were not manifest in this small series of patients in concordance with results from similar recent European series. Dedicated liver MRI screening along with quarterly liver profile and platelet counts to monitor for NRH should be offered to patients on long term therapy.

Disclosure of Interest None Declared.

\section{PWE-082 W/THDRAWN BY AUTHOR}

\section{PWE-083 THE DEVELOPMENT OF A STRATIFIED MODEL OF FOLLOW UP CARE FOR ADULT PATIENTS WITH INFLAMMATORY BOWEL DISEASE}

doi:10.1136/gutjnl-2013-304907.371

1."K Kemp, ${ }^{2} \mathrm{~J}$ Griffiths, ${ }^{3} \mathrm{~S}$ Campbell, ${ }^{2} \mathrm{~K}$ Lovell. 'School of Nursing/Gastroenterology, University of Manchester/Manchester Royal Infirm, Manchester; ${ }^{2}$ School of Nursing, University of Manchester, Manchester; ${ }^{3}$ Gastroenterology, Manchester Royal Infirmary, Manchester, UK

Introduction There is concern in the UK that services for pts with long term conditions are not orgnised to promote independence with silo working in primary and secondary care with reactive services. These may be brought together formally through the development of model of care. Utilization of current out-pt spaces to regularly review stable pts is inappropriate and is challenged by commissioners. The question remains as to what models of follow up (FU) are we able to offer pts which are acceptable and feasible. The aim of this study was to develop an integrated, acceptable, modern model of FU care for pts with IBD.

Methods Using the MRC Framework for complex interventions, 24 IBDs (18 pts had CD, $6 \mathrm{UC}$, age range 27-72 years, disease duration range $2-40 \mathrm{yr}$ ), 20 purposively selected GPs from NW England, and 3 IBD Nurses (specialist, advanced practitioner, consultant nurse) were interviewed. Participants were asked about the role of FU in IBD, experience of FU patterns, service delivery, other models of FU. Thematic analysis was undertaken using NVivo 9.0. Analysis of 3 groups of interviews were synthesised by a Cons Gastroenterologist, patient, GP, IBD Nurse, to develop the model of FU care. 


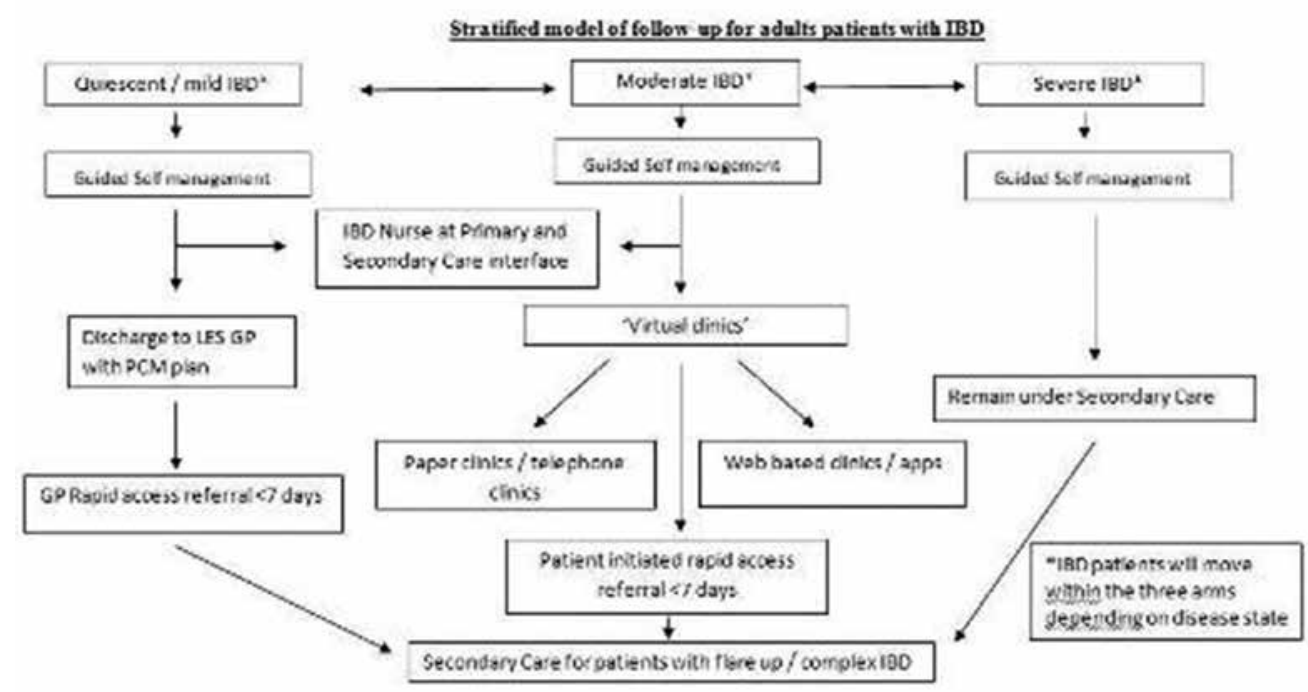

\section{Abstract PWE-083 Figure}

Results There were similarities between 3 groups of interviews. Pts did not want to be seen when well, GPs wanted more involvement in care and there is scope for an IBD outreach nurse at interface of primary/secondary care. Discharging quiescent pts into enhanced GP care, to ensure equitable treatment, was acceptable to all, as was the concept of 'virtual' clinics. Patients would initiate self referral within the virtual arm whilst pts under GP care would be referred by GP. Pts would be referred as a rapid FU $<7$ days and not as a new pt tariff. Complex IBD patients would remain under secondary care. Patients will move across the 3 arms depending on disease.

Conclusion This study provides an acceptable integrated model of FU for pts with IBD. It takes into account UK policy to reduce inappropriate $\mathrm{FU}$, with emphasis on self management and integrating care, placing the pt closer to home, with secondary care emphasis on complex pt management.

Disclosure of Interest None Declared.

\section{PWE-084 WHOLE BLOOD MRNA EXPRESSION PROFILING OF CROHN'S DISEASE IN THE CERTIFI USTEKINUMAB STUDY DISCRIMINATES CLINICAL SUBTYPES}

doi:10.1136/gutjnl-2013-304907.372

${ }^{1} \mathrm{~K} \mathrm{Li},{ }^{1} \mathrm{C}$ Brodmerkel, 'S Telesco, ${ }^{1} \mathrm{~K}$ Ma, ${ }^{1{ }^{*} \mathrm{C}}$ Gasink. 'Janssen. R\&D LLC, Spring House, United States

Introduction Objective markers of Crohn's Disease (CD) activity have been sought as diagnostic, phenotypic, prognostic and disease activity markers. Complications such as stricture and fistula and characteristics such as TNF-antagonist responsiveness have been suggested as discreet mechanistic CD subtypes. This study explored the ability of genome wide expression profiling in whole blood to differentiate CD sub-populations.

Methods In the previously reported Phase $2 b$ ustekinumab CERTIFI study of patients with moderate to severely active CD who had failed or were intolerant to TNF-antagonists, whole blood samples were collected from a subset for mRNA expression profiling using Affymetrix HG-U133+ PM arrays. Baseline expression profiles were compared between patient sub-groups characterised by defined baseline disease attributes; and compared with those from samples obtained independently from healthy subjects. Expression modulations of $>+/-1.5 x$ and false discovery rate (FDR) $p$-value $<0.05$ were considered significant.
Results Patients ( $\mathrm{n}=204)$ with moderate to severe CD had significant expression modulations in 1725 transcripts in the whole blood compared with healthy subjects $(n=49)$, including genes involved in inflammatory response and connective tissue disorders. A panel of 20 transcripts (including GAB2 and IL18R1) discriminated patients with only colonic $(n=49)$ vs. strictly ileal $(n=60)$ disease involvement. Significantly different expression modulations of 169 , 321, and 151 transcripts, respectively, were identified in patients with high baseline CRP $(>10 \mathrm{mg} / \mathrm{dL}, \mathrm{n}=97)$, faecal calprotectin (>850 mg/g, $\mathrm{n}=80$ ) or lactoferrin $(>100 \mathrm{mg} / \mathrm{g}, \mathrm{n}=89$ ) compared with patients with low baseline CRP $(<3 \mathrm{mg} / \mathrm{dL}, \mathrm{n}=45)$, faecal calprotectin ( $<250 \mathrm{mg} / \mathrm{g}, \mathrm{n}=58)$, or lactoferrin $(<100 \mathrm{mg} / \mathrm{g}, \mathrm{n}=107)$. As expected, patients with high baseline CRP, faecal calprotectin, or lactoferrin had elevated gene expressions in inflammatory pathways such as IL- 6 and acute phase response signalling. In contrast, gene expression profiles did not differentiate between patients with different durations of disease (long $[>15 \mathrm{yrs}]$ vs. short $[<5 \mathrm{yrs}]$ ); prior treatment response (Primary responder vs. non-responder) and treatment history (number of TNFs failed); and the presence or absence of complications (stricture/stenosis, fistula).

Conclusion Genome-wide expression profiling of peripheral blood samples provides the understanding of CD at the molecular level in circulation. This is a new, non-invasive method that can be used to identify systemic markers of local pathological alterations in $\mathrm{CD}$ and to discriminate clinically between different $\mathrm{CD}$ sub-types.

Disclosure of Interest K. Li Employee of: Janssen R\&D, LLC, C. Brodmerkel Employee of: Janssen R\&D, LLC, S. Telesco Employee of: Janssen R\&D, LLC, K. Ma Employee of: Janssen R\&D, LLC, C. Gasink Employee of: Janssen R\&D, LLC

\section{PWE-085 CT ENTEROGRAPHY REMAINS A VALUABLE TOOL FOR THE ASSESSMENT OF CROHN'S DISEASE}

doi:10.1136/gutjnl-2013-304907.373

1,"K L White, 'C A Boyd, ${ }^{2} \mathrm{M}$ Sapundzieski, 'J K Limdi. 'Gastroenterology; ${ }^{2}$ Radiology, Pennine Acute Hospitals Trust, Manchester, UK

Introduction Advances in the immunopathogenesis of inflammatory bowel disease (IBD) coupled with bolder definitions of disease control have led to increasing reliance on imaging to characterise inflammation beyond the reach of the endoscope. Clinical activity 